\title{
Guest Editorial: Scale-Space and Variational Methods
}

\author{
Bart M. ter Haar Romeny • Christoph Schnörr
}

Published online: 6 June 2013

(c) Springer Science+Business Media New York 2013

This special issue highlights some recent contributions to scale-space theory and variational methods for mathematical and computational image analysis.

A novel approach to dictionary learning for representing large data sets by sparse linear combinations in terms of so-called coresets, is presented in the paper "Learning Big (Image) Data via Coresets for Dictionaries" (DOI 10.1007/s10851-013-0431-x). Bounds on the size and the approximation error of optimal dictionaries are derived, and parallel or online versions of the learning algorithm may scale up to very large data sets.

The issue of scale selection for accurate large-scale intersubject image registration is addressed in the paper "Sparse Multi-Scale Diffeomorphic Registration: the Kernel Bundle Framework" (DOI 10.1007/s10851-012-0409-0). The corresponding variational formulation and evolution equations constitute an exiting recent development related to research on diffeomorphism groups, shape theory and computational anatomy.

The paper "Exact Histogram Specification for Digital Images Using a Variational Approach" (DOI 10.1007/s10851012-0401-8) studies the problem of transforming a given digital image (i.e. with quantized pixel values) so as to attain a pre-specified histogram. To cope with this highly ill-posed problem, a variational approach is devised that additionally preserves characteristics of the input image.

A geometric approach to invariant and structure preserving image processing is considered in "Morphological and Linear Scale Spaces for Fiber Enhancement in DWMRI" (DOI 10.1007/s10851-012-0387-2). Authors develop a theory of morphological and linear scale spaces on subRiemannian manifolds related to the Euclidean group so as to achieve crossing-preserving enhancement of fibers in diffusion-weighted MRI.

Finally, the paper "Statistical Multiresolution Estimation for Variational Imaging: with an Application in PoissonBiophotonics" (DOI 10.1007/s10851-012-0368-5), introduces a spatially-adaptive approach to image restoration in terms of so-called statistical multiresolution estimators, that minimize a $\ell_{\infty}$-type data likelihood together with a convex regularizing term. A key property of the approach concerns an automatic selection rule for weights that balance the influence of these two terms.

We hope the reader will enjoy these contributions to mathematical imaging and vision.

B.M. ter Haar Romeny

Eindhoven University of Technology, Eindhoven,

The Netherlands

C. Schnörr (凶)

Heidelberg University, Heidelberg, Germany

e-mail: schnoerr@math.uni-heidelberg.de 